\title{
Neuroscience and Digital Learning Environment in Universities: What does current research tell us?
}

\author{
Betsy Ng \\ National Institute of Education, Nanyang Technological University \\ betsy.ng@nie.edu.sg \\ Aloysius Kian Keong Ong \\ National Institute of Education, Nanyang Technological University
}

\begin{abstract}
The purpose of this article is to offer insights into current understanding of digital learning environments (DLEs) from a neuroscientific perspective. Cognitive neuroscience methods are increasingly applied in educational research to examine the neural underpinnings of learning. As such, neuroscientific evidence can play an important role in advancing current knowledge base from the existing self-reported data and behavioural measures in the field of educational technology. In this paper, we focus our review of neuroscience research on DLEs that can potentially transform the way we view learning and instruction. We discuss recent empirical studies done on DLEs using common cognitive neuroscience methods which included eye tracking, electroencephalography (EEG), and functional magnetic resonance imaging (fMRI). We offer recommendations for future applications of neuroscience methods in behavioural research within DLEs.
\end{abstract}

Keywords: Digital learning environment, behavioural, learning, neuroscience

\section{Introduction}

Educational technologies are emerging as an important instructional means to facilitate student engagement and self-directed learning in universities (O’Flaherty \& Phillips, 2015). For instance, traditional teaching has been combined with digital learning environments (DLEs) to offer new opportunities for student-centered learning and to facilitate students' knowledge construction. Such a form of blended learning is becoming commonplace in universities and constantly undergoing design changes to adapt to varied higher institution learning settings (O’Flaherty \& Phillips, 2015). Existing research studies in higher education have shown that students are more likely to engage when they learn using DLEs (Bryson \& Hand, 2007; Hockings, Cooke, Yamashita, McGinty, \& Bowl, 2008). However, the understanding of how students actually learn and engage in DLEs is less informed.

Recently, there has been increased research attention on addressing issues of learning and instruction using neuroscience methods and behavioural approaches. One key area of learning explored using the neuroscientific approach, is on the learners' cognitive processes during reading and learning (Grabner et al., 2009; Yoncheva et al., 2010). Functional neuroimaging techniques, such as fMRI, detect increased blood flow that is thought to be coupled to increased neuronal activation during a cognitive process, and measure a learner's neuronal activity based on a given task (e.g., Prat \& Just, 2011). Current fMRI studies explore cognitive activities of undergraduates in reading and comprehension (e.g., Henderson, Choi, Lowder, \& Ferreira, 2016). The application of neuroscience methods in educational research potentially allows researchers to study student 
engagement level such as how students actually perform and engage in online learning tasks (Ansari \& Coch, 2006). Together with behavioural measures, neuroscientific data may therefore provide important evidence of students' deep engagement levels of learning during an online activity. This paper aims to examine how cognitive neuroscience methods are used with behavioural measures to inform evidence-based research in the field of educational technology. This review paper provides a focused theme on some current advances and perspectives on the application of cognitive neuroscience to understand student learning in DLEs at university level.

\section{Digital Learning Environment}

The emphasis of self-directed learning in universities is fundamental to Knowles' (1970) view of developing an environment that is conducive to individual orientation to learning and needs. In light of this, the seamless integration of technology into instruction and learning has offered potential powerful DLEs to facilitate and advance individual learning through inquiry and collaboration. Rapid technological developments have also resulted in extensive research on the implementation of information and communication technologies (ICT) as well as digital tools in schools and higher learning institutions (Säljö, 2010). For example, DLE is a key technological development that has been widely researched. Broadly, DLEs can be defined as the use of technology to support student learning, teaching and studying activities (Anderson, Love, \& Tsai, 2014). The model of learning in DLEs is consistent with that in the learner-centred and the constructivist model, and both emphasize the construction of knowledge. Common examples of DLEs include computer-supported learning, mobile learning and multimedia learning with representations such as text, graphic, video and animation to enhance student learning (Anderson et al., 2014; Chang \& Linn, 2013). In this paper, DLE also includes an e-learning platform, webbased and multimedia instruction.

DLEs are an increasingly important mode of instructional delivery in higher education as a means to promote personalized and engaging learning experiences (Dahlstrom \& Bichsel, 2014). The availability of broadband access affords many courses the opportunity to be taught online, and universities are increasingly turning to the use of DLEs to promote online learning. This is evident in at least two DLEs: Learning Management System (LMS) and Massive Open Online Courses (MOOCs). The LMS is not only a widely used administrative system for course management, but it also functions as a DLE. For example, online teaching and distance education are delivered through the LMS to provide students with a personalized and engaging experience (Dahlstrom \& Bichsel, 2014; Dahlstrom, Brooks, \& Bichsel, 2014). MOOCs are low fee or free web-based instructional modules offered by universities or educational institutions (e.g., Coursera). MOOCs can have a very high enrolment; however, the level of learner engagement in the DLE is often less encouraging (Dahlstrom \& Bichsel, 2014). Success factors for DLEs have been mainly attributed to student learning satisfaction, personal traits and attitudes (Stokes, 2001). Recent research, however, suggests that cognitive neuroscience can further our understanding of how and why students learn in DLEs. For instance, a learner's brain areas that are activated during an online task can be tracked using neuroscience methods.

\section{Neuroscience and Education}

Cognitive science with learning, coupled with neuroscience methodologies have created a new domain in educational studies, that is, cognitive neuroscience. Cognitive neuroscience has been 
extensively studied in the area of psychology research (Stewart, 2015), but there is still limited research in the field of DLEs. Past research has delved into motivational, cognitive and emotional dimensions of learning, as well as cross-cultural differences in reading and mathematical reasoning (Montague et al., 2006; Siok et. al., 2004; Tang et al., 2006). More recent works explore real-time cognitive and neurophysiological phenomena because psychological behaviour has neural underpinnings which could influence physiological activity (Botvinick \& Braver, 2015). Cognitive neuroscience research findings provide evidence that explains and predicts human learning (Bassett \& Mattar, 2017). The interdisciplinary research of cognitive neuroscience and educational psychology may contribute to a neuro-educational theory, informing the best practices in various learning aspects including DLEs. Three common cognitive neuroscience techniques are evident from the literature, and these techniques include eye tracking, EEG and fMRI. Eye tracking studies attentional focus during a learning activity; EEG measures the electrical activity in the brain during a cognitive task; while fMRI estimates the changes in brain activity associated with changes in blood flow during a learning process. Conceptual change refers to "removal or restructuration of prior knowledge” (Masson et al., 2012, p. 29), and fMRI is used to study conceptual change in science learning and to test new research hypotheses, leading to new interpretation of the nature of conceptual change.

The utility of neuroscience findings for educational research is a current debate. Diamond and Whitington (2015) claimed that the gap between "what we know and what we do" can be bridged by neuroscience findings. However, findings from neuroscientific research typically draw on small sample sizes and are not generalizable. Moreover, there has been a lack of neuroscientific research in schools and university settings. It is therefore not clear how neuroscience findings can actually bridge the gap between theory and practice. Nevertheless, we recognize that existing selfreported measures applied in behavioural research are limited in understanding the cognitive processes of learners in a DLE. To extend our current knowledge base in this field, it is important to explore how neuroscientific approaches and findings could corroborate data from behavioural research, and inform about learning during online activities or courses. Neuroscience approaches may be used to research DLEs. This paper aims to show how findings from neuroscience approaches can complement behavioural research to enhance our understanding of learning supported by educational technology. We discuss three common cognitive neuroscience methods, namely, eye tracking, EEG and fMRI, as well as the use of these methods in advancing the understanding of student learning and skills in DLEs at universities. To understand the potential translation of neuroscience into DLEs, we review 20 recent empirical studies based on the neuroscience methods in the field of educational technology at university level.

\section{Eye tracking}

Eye movement research relates not just to the study of perceptual systems, but also relates to the study of neuroscience (Richardson \& Spivey, 2004). Eye tracking is one of the common neuroscience techniques used in educational research (Anderson et al., 2014). This method involves the study of eye movements which comprise "a series of fixations and saccades while reading information or viewing scenes" (Lai et al., 2013, p. 92). Fixation is defined as "a relatively stable state of eye movement" while saccade refers to "rapid eye movement between two consecutive fixations". Eye tracking provides both quantitative and qualitative analyses of the subject's gaze (Popa et al., 2015) by capturing data related to individual interest, level of attention and visual attention during a learning activity. The main method used for recording eye movement 
is by electrooculography (EOG). Currently, eye tracking has been widely used in psychologyrelated fields to study basic cognitive processing during reading and information processing (e.g., Rayner, 2009). One notable focus area is the visual attentional processes during multimedia learning (e.g., Jamet, 2014; Yang et al., 2013).

A literature search shows 12 recent empirical studies that investigated the pedagogical and cognitive aspects of DLEs at university level. From our review, these studies focused on two broad areas of learning and instruction, namely instructional aspects and learner attributes, respectively.

\section{Instructional Aspects}

Instructional aspects refer to the pedagogical components or approaches that influence learning. We identified four categories of instructional aspects from the students: cueing conditions, pace of instruction, instructional task, and type of devices.

\section{Cueing conditions}

Studies that addressed the effect of different cueing conditions on visual attention and cognitive load during learning included: Boucheix and Lowe (2010), Jamet (2014) and König et al. (2010). These three studies specifically examined visual attention and text comprehension, but their findings revealed mixed conclusions. Boucheix and Lowe (2010), for example, showed that cueing conditions were positive on undergraduates' comprehension and orientation of attention during learning of the animated piano mechanism (i.e., the mechanism of note production when a key on the piano keyboard is pressed). Three cueing conditions, namely an arrow cue condition, a spreading-colour cue condition and a no-cue condition, were studied on 57 psychology undergraduates. Both arrow cue and spreading-colour cue conditions had greater fixation numbers and durations than the non-cued condition, indicating direct attention guiding helped to facilitate learner processing and efficient comprehension of the animated piano mechanism.

Jamet (2014) also found that attention guiding had improved oral and visual information processing during multimedia learning. She worked with a sample size of 32 psychology undergraduates and her findings revealed a positive effect of visual sources on the learner retention of signalled information within a DLE. However, König and colleagues (2010) conducted an experimental study of three types of cueing conditions (i.e., single cue, multiple cues and no cue) in the context of cardiovascular system (i.e., learning about the functions of human cardiovascular system) and found no effect of cueing on visual attention and cognitive load. Their findings also indicated no effect of cueing on the comprehension of computer-based cardiovascular contents. König and colleagues suggested that the short duration of cueing might be the limiting factor to explain the findings. The difference in methodology between the two studies is the duration of the animations. Jamet’s (2014) multimedia learning involved a total duration of 420 s, whereas König and colleagues (2010) used a computer presentation with a 132-s animation.

\section{Pace of instruction}

Schmidt-Weigand et al. (2010) and Meyer et al. (2010) focused on the effect of the pace of instruction on the learner within multimedia learning settings. Schmidt-Weigand et al. (2010) found that the pace of instruction positively correlated with visual information processing among undergraduates in multimedia learning. Using eye tracking, they examined how undergraduates 
split their attention in multimedia learning and how their visualisation changed with the pace of instruction (i.e., fast, medium and slow). While the researchers concluded that visual attention changed with pace of instruction, their results were limited to a specific learning content and small sample size $(N=31)$. In another related study, Meyer et al. (2010) examined whether presentation speed of an animation would influence the patterns of eye movements on 74 undergraduates from education and psychology in a German university. The animation was also limited to a specific learning content - a presentation on four-stroke internal-combustion engine. However, Meyer and colleagues found that the presentation pace (fast-to-slow vs. slow-to-fast) had no strong effect on the duration of eye fixations. In addition, the presentation pace had an impact on comprehension of micro- and macro-knowledge, but no strong effect on overall comprehension. Their findings revealed mixed evidence on how presentation pace affects students' learning with animations. The inconsistency in results could be due to the constraint design of the animation, which relates to how instructional design of a learning environment may impact student learning.

\section{Instructional task}

A few of the reviewed studies addressed the effect of instructional task on visual attention. Tsai and colleagues (2012) studied the effects of 6 male university students' visual attention on an online science problem. The content of the science problem was debris slide hazard with multiple choices for student selection. Students spent time inspecting the relevant factors in order to predict the debris slide hazard. Their study found that successful problem solvers paid more attention to the chosen options and relevant factors than unsuccessful ones. Similarly, Chen and Yang (2014) investigated 20 university students' cognitive processes in an online spatial problem solving and science concept learning. Eye movement patterns correlated with spatial problem solving but not concept performance, suggesting that spatial problem-solving task might not be specifically designed to reflect the science concept. Susac and colleagues (2014) investigated 40 undergraduates' strategies in simple equation solving by eye tracking. Eye tracking data in addition to the questionnaire reports provided information about learners' efficiency in solving simple algebraic equations and strategies used during equation solving. Their findings revealed an inverse relationship between number of fixations and efficiency in problem solving. This means that the number of fixations was reduced when students' efficiency in equation solving had increased, suggesting efficient strategies employed during equation solving.

\section{Type of devices}

Molina et al. (2014) investigated the use of various devices in DLEs. They assessed the learning performance of 20 undergraduates on their use of different devices (mobile phone vs. tablet vs. desktop computer) to access learning materials. The researchers used an eye tracker, Tobii X60, to study the learners' eye movements, which included the duration of visualisation. The time spent in visualisation was related to understanding and assimilating the learning contents online. They found that the type of devices for accessing the learning materials influenced the learner performance. Their findings showed that desktop and tablet were more suitable to access and visualise learning materials than mobile phone because of less cognitive load. Molina et al. (2014) also found that learners were more motivated to access learning materials with a tablet than a desktop. 
Ng and Ong

\section{Learner Attributes}

Learner attributes refer to the cognitive aspects of learners that play a role in shaping learning. The categories included content knowledge and working capacity.

\section{Content knowledge}

Two studies focused on the correlation between students' content knowledge and visual attention in DLEs. Canham \& Hegarty (2010) examined the possible interactions between task-relevant versus task-irrelevant information and content knowledge on 16 undergraduates from an introductory psychology course on the content of meteorology. They found that students with prior meteorological knowledge had a greater visual attention on the relevant information than the irrelevant information in weather maps during the tutorial. The authors concluded that taskrelevant information on display played an important role in student comprehension of meteorology and that the design should not provide more information than needed for the novice learners.

In another study, Yang et al. (2013) used eye tracking to examine 21 university students' visual attention distributions during a PowerPoint multimedia learning experience. Based on an instructional design, 10 university students formed the earth-science (ES) group while 11 were assigned to the non-earth-science (NES). Students with ES content knowledge (i.e., ES group) had greater attention distributions, higher relevant knowledge and higher memory test scores than NES students. These findings suggest that students with relevant content knowledge about ES had greater visual attention and scored better than those in the NES group.

\section{Working capacity}

The study conducted by Ariasi \& Mason (2014) examined the working capacity of learners. The researchers used eye-tracking to investigate how 63 undergraduates processed with different working capacity while reading different types of scientific texts, refutation text versus nonrefutation text in the domain of physics. Their results revealed that the higher the readers' capacity to process and temporarily store information while reading, the greater their knowledge gains. The findings suggest that eye tracking is able to evaluate the learner working capacity during reading in a DLE.

Our review from these 12 empirical studies shows that the eye tracking offers a quantitative and objective measure of visual attention when reading texts and processing information in DLEs. Of these studies, eye movements were used to understand the interactions between visual attentional processes and learning outcomes such as contents comprehension and problem solving. However, findings from the studies are mixed, and it is still premature to draw conclusions on the interactions between visual attentional processes and learning outcomes from eye tracking. We next discuss studies on higher education learning informed by the EEG.

\section{Electroencephalogram (EEG)}

The principle of EEG technology is the placement of electrodes or channels on the head to measure scalp potentials (Hames \& Baker, 2013). These scalp potentials represent "the linear superposition of electric dipoles within the brain" which are distributed throughout the brain regions. EEG signals reveal the electrical activity in relation to a cortical response. The electrodes detect EEG 
signals that can then facilitate the understanding of student brainwave in action and student engagement during a cognitive task. Our literature search shows 4 recent empirical EEG studies on student emotion and learning experience in both computer-based environment and classroom.

EEG is commonly used with behavioural measures to examine emotional states of the learner in an online learning system (Shen, Wang, \& Shen, 2009). EEG signals in relation to emotions are analysed using the following frequency bands (Shen et al., 2009): delta (0-4 Hz), theta (4-8 Hz), alpha (8-13 Hz), low beta (13-20 Hz), and high beta (20-40 Hz). Each frequency range is associated with different levels of arousal. For instance, alpha waves are related to calmness while high beta frequencies are associated with high levels of arousal and may lead to experiencing anxiety (Shen et al., 2009). According to Russell (1980), emotions are viewed as a combination of arousal and valence (negative to positive). Recent studies have shown that affective characteristics play an important role in computer-based learning and e-learning (e.g., Wang \& Hsu, 2014). Liu et al. (2011) conducted a computer-based music experiment of 10 subjects with ages ranged from 23 to 35 . They found that 5 subjects had the right hemisphere exhibiting greater EEG activity during the experience of negative emotions, while 4 subjects had the left hemisphere showing higher EEG activity during positive emotions (Liu et al., 2011). This finding suggests that individual differences may influence the processing of emotion by brain and frontal lateralization exists with individual differences.

Shen and colleagues (2009) used an affective e-learning model to recognise an undergraduate's emotion with brainwave signals, in the context of the student's chosen subject. Their results showed a close relationship between emotion and brainwave signals, suggesting that affect plays an important role in engagement. Such affective model is related to student cognitive skills, goals of learning and interaction with the learning system (Shen et al., 2009). EEG findings may predict student emotions in learning and explore how student emotions evolve during the learning process in a DLE.

The utility of the EEG system can facilitate student learning by improving interest and attention in the areas of neurocognitive psychology (Stewart, 2015). Stewart examined the utility of EEG classroom demonstration on undergraduates taking introductory psychology course. Participants who received the EEG demonstration performed better on a quiz testing the lecture materials. Steward's (2015) findings suggest that EEG technique served the purpose of improving student engagement and increasing attention levels, accompanied by increased quiz scores. Emotion detection from brainwave signals could thus provide feedback to teachers, improving student learning experiences.

With regard to learning outcomes, Wang and Hsu (2014) examined the influence of learner flow experience on enjoyment and focused attention. Their results demonstrate that increased flow experience improved learning performance and learning satisfaction in a computer-based instructional environment. Likewise, Chen and Wu (2015) assessed the effects of different online video lecture formats (i.e., lecture capture, picture-in-picture and voice-over) on student sustained attention, emotion, cognitive load, and learning performance. All three video types significantly promoted learning performance, with lecture capture and picture-in-picture better than voice-over format.

Recent studies have shed some light on the current mobile EEG technologies. Mobile wireless EEG devices are available for detecting and analyzing EEG signals of the frontal lobe, which provide student attentiveness during learning (Liu, Chiang, \& Chu, 2013). EEG is a useful neuroscience method for recording neural activity of a subject. Nevertheless, EEG does not 
provide a considerably fine spatial resolution due to a greater temporal resolution than fMRI, which is described in the next section.

\section{Functional Magnetic Resonance Imaging (fMRI)}

Over the past decade, neuroscientific technologies such as fMRI have significantly improved the understanding of brain structure and function. For instance, language involves syntactic processing that is shown by the activation of the core network of left inferior frontal gyrus, left posterior middle and superior temporal gyrus (Weber, Christiansen, Petersson, Indefrey, \& Hagoort, 2016). When learning a new language, the repetition of novel word orders would lead to fMRI repetition effects, indicating presentation of syntactic structures (word orders) and repetition enhancement (increased cortical activation). Existing fMRI research in education focuses on the cortical systems that represent syntactic and semantic components of the human language (Henderson et al., 2016). However, fMRI in education, particularly in online learning environment remains less informed. From our literature review, we found 4 studies related to the areas of language reading and comprehension. We did not find any study in cognitive process and learning of university students in the context of online environments. We reviewed the empirical studies on language processing and neurocognitive mechanisms of university students.

First, neuroscience research has explored representation and processing of syntactic categories (e.g., noun and verb). Henderson and his colleagues (2016), for example, used a combined approach of eye tracking and fMRI to examine the cortical implementation of syntactic computations during native language comprehension on 40 native speakers of English. Their results revealed activation in cortical regions that are associated with prediction in language. The combined approach is an alternative method of testing the theoretical questions in language processing during reading. Henderson et al.'s work showed how the neuroimaging technique was able to measure the neural foundations of syntax and its effects on comprehension.

Second, Zhao and colleagues (2012) used fMRI to study how different language systems had different syntactic representation and lexical processing. Fifteen native alphabetic languagespeaking university students who learnt Chinese as second language (L2) for at least one year exhibited stronger activation in the right lingual gyrus and right fusiform gyrus than the 15 native Chinese speakers. The stronger brain activation in L2 learners suggests that they required accommodation and assimilation patterns for reading of Chinese characters.

Third, Weissberger et al. (2015) examined the relationship between linguistic and nonlinguistic executive control on 21 bilingual (English-Spanish) undergraduates with a 2 (language vs. colour-shaped tasks) $\times 3$ (switch, single and stay trials) experimental design. Participants were cued to switch between tasks (i.e., switch), perform one task (i.e., single) and perform the same task as the preceding trial (i.e., stay). Their fMRI results showed a considerable overlap in brain regions underlying language and colour-shaped task on switch trial versus single and stay trials. On the contrary, there are fewer overlapped brain regions between tasks on stay trial. These findings suggest that a bilingual participant requires some cognitive demands for successful language switching and communication.

Finally, Prat and Just (2011) used Scanning Probe Microscopy 2 (SPM2; Wellcome Department of Imaging Neuroscience) to examine the distribution of brain activation during sentence comprehension as a function of cognitive capacity and individual verbal working memory. Collection of fMRI data was done by axial slice acquisition timing, motion-corrected and normalized to the Montreal Neurological Institute (MNI) template. Based on fMRI data, high- 
capacity readers exhibited increased activation in both striatum and prefrontal regions with increasing syntactic complexity than low-capacity readers, indicating greater neural adaptability to changing linguistic demands.

Current evidence about learning in higher education relies heavily on questionnaires which may be constrained by self-reporting bias (e.g., Wu, 2015). As discussed in the abovementioned studies, brain imaging offers insights into the nature of cognitive processes involved in academic tasks (Masson et al., 2012). However, a major drawback of the fMRI scanner is the cost. The fMRI requires a very expensive set-up, including high costs for renting the scanner and hiring the technical experts. Moreover, fMRI scans are expensive and significant funds are needed for fMRI data collection. Expensive software packages are also needed to interpret the fMRI data (Steinmetz \& Atapattu, 2010). Furthermore, the fMRI scanner permits only one subject for each scan which is not cost-effective. Nevertheless, fMRI data may help us establish a predictive, broad framework for a neuro-educational theory to inform educators about human learning and brain neural networks. Finally, we need to consider the implications of cognitive neuroscience for improving or refining our practices in learning and instruction.

\section{Implications for Research and Practice}

Our review of current empirical studies highlights the following trends in educational neuroscience: (1) eye tracking focuses on attention studies related to computerised activities and e-learning (e.g., Molina et al., 2014); (2) EEG measures student engagement level in an online learning system and cognitive load during an online lecture (e.g., Chen \& Wu, 2015); and (3) fMRI research focuses on computerised cognitive tasks such as reading and working memory (Zhao et al., 2012). We argue that these neuroscience methods (eye tracking, EEG and fMRI) enhance our understanding of human learning in DLEs in two ways. First, these neuroscience methods open up a new perspective of looking at student learning and cognitive processes that can stimulate new research hypotheses. Second, findings from these neuroscience methods revealed that neuroscientists and educators need to work more closely together to translate neuroscience research into education paradigm, which may help to bridge gaps between neuroscience and education.

There are still gaps in the current literature of neuroscience approaches and student experience in DLEs that need to be addressed. One area is the limited understanding on how pedagogical aspects of DLEs can improve student learning, as most DLE studies tend to neglect this concern and focus on course evaluation or learning outcomes. Another area is the lack of understanding of student engagement and learning process for online courses.

EEG has the potential to inform whether students enjoy from online learning or they find it too challenging. Yet, studies in this area particularly in MOOCs have not received attention. EEG data might also help to explain student engagement and dropout rate in MOOCs, which is high. Users do not continue the online courses after enrolment (Freitas, Morgan, \& Gibson, 2015). Perhaps, students who fail to engage in the course are also dropping out (Henrie et al., 2015) may not be an ability issue. It may be due to a lack of motivation or other reasons. EEG can be used to track student engagement level during a learning activity. For example, student emotions can be collected and interpreted as frustration or boredom by the computer (Gil, Virgili-Gomá, García, \& Mason, 2015).

Existing fMRI research in neuroscience education tends to focus on the cortical systems that represent syntactic and semantic components of the human language (Henderson et al., 2016). Our review of current literature suggests that there is much research attention on the patterns of 
information processing of university students in language comprehension and reading using fMRI (e.g., Henderson et al., 2016; Prat \& Just, 2011). Such studies typically employed cognitive tasks in the form of block design for presenting stimuli within the scanner. As only one subject is scanned each time, fMRI can reveal individual differences in verbal working memory capacity and information processing capability with a particular task or class of tasks. By using the scanner environment, we are able to obtain individual neural substrates in the time-course of e-learning in relation to behavioural performance at multiple time points. However, fMRI has one main methodological limitation - the participant in the scanner provided responses to both tasks using button presses that were flashlight monitored by the scan operator, which could lead to inaccuracy of responses. Neural adaptability to changing task demands may help us understand the learner cognitive ability in coping with the linguistic demands in DLEs. Research in areas of cognitive and learning processes in DLEs may need more work.

Perhaps the key challenge to the work of measuring student engagement and motivation in DLEs is the lack of consistency among model, operationalisation and instructional tool. Although most neuroscience studies focused on cognition and brain activity, the recent studies explored how brain imaging relates to motivation (e.g., Lee \& Reeve, 2012). For example, a neurotransmitter known as dopamine is associated with cognition and reward seeking during the decision making process (Kanwal, Jung, \& Zhang, 2015). The peak of dopamine release in the brain could relate to an individual's decisions that are more reward-based, linking to extrinsic motivation. Neuroscience thus brings new ways of understanding student engagement. Furthermore, neuroscience and educational methods may potentially model the delivery of online learning to students and improve their learning experience.

DLE is attributed to social, cognitive and teaching presence (Kozan \& Richardson, 2014) because it involves complex and educational experience. Brain imaging techniques may provide insights into the activation of brain cortical area central to an online task and emotional activation of amygdala due to learning anxiety. The understanding of such concepts is currently limited in the areas of online and blended learning. DLE is unique to the learning of concepts across diverse learners, such that learners may experience different levels of emotion during learning. Understanding the levels of emotion by brain imaging may lead to a new research hypothesis and support the existing findings. Such effort to bridge student digital learning and cognitive process could reveal new research findings and further develop the basis of empirical findings related to education.

Neuroscience may be able to provide specific evidence into certain groups of learners who do not benefit sufficiently from the DLEs. The application of EEG and fMRI during a decision making or problem-solving process may provide insights about why some students fail to learn within the DLEs. As such, neuroscientific evidence may provide insights into the constraints of the learning brain and help to explain why some learning environments fail to improve student performance. Neuroscientific data may also offer specific knowledge required to design powerful DLEs in particular to academic content areas. Such evidence-based research is useful to inform online learning and DLEs.

Despite the abovementioned benefits of neuroscience research, there are concerns about the interpretation of neuroscientific facts and selection of relevant findings to support behavioural methods. There are also challenges in designing neuropsychological studies as they require interdisciplinary effort and team. First, to integrate neuroscience methods into behavioural research, we have to collaborate across multi-disciplines, in particular the neurocognitive scientists, learning designers, educational researchers, and educators. The collaborators need to be clear of 
one another's role and able to connect the fields of neuroscience and education. It is important to note that neuroscience methods may not provide direct evidence about the key educational considerations such as context (Varma, McCandliss, \& Schwartz, 2008). Hence, when contextualising contents in a DLE, it is necessary to work with the learning designers or the expertise in instructional design. Second, neuroscience should not replace education research. Instead, neuroscience methods should be used as a complementary means to support findings of education research. This is because neuroscience approaches are not able to provide a causal-effect solution. Therefore, neural correlates are used to support data from the behavioural measures. We should be mindful of such limitations and employ neuroscience methods in a complementary manner. Finally, the pragmatic reason is neuroscience methods are expensive to apply. Although neuroscience methods may resolve some of the limitations within education, policy-makers and stakeholders should practise a cautious optimism. To an extent, bringing neuroscience methods into educational research may appear too upstream approach to the policy-makers who provide research funding to education. Unless there are government agencies who advocate for such research, educationally relevant neuroscience proposals might attract additional research funding grant.

To sum, the utility of neuroscientific techniques enables us to focus on the learning process rather than the learning outcomes. The neuroimaging findings are able to give us a better understanding of the brain, indicating the specific areas of brain activation which in turn could correlate with the behavioural results. As such, neuroimaging findings might support the selfreported data and explore brain regions with neural activation in relation to changes in performance during an online activity. Given the potential payoff of integrating neuroscience methods into the field of educational technology, we would be able to understand the "why" and "how" students learn in DLEs. We propose that neuroscience research should be anchored in a multi-disciplinary collaboration. This way, research can better inform practice. With neuroscience evidence informing the different aspects of learning and instruction in particularly DLEs, future research should move into an interdisciplinary effort to look into intervention studies.

\section{Conclusion}

The present paper provided a discussion of the use of neuroscience methods (corroborated by behavioural measures) to study brain mechanisms related to learning and instruction within the field of educational technology. We found that eye tracking is most widely applied in cognitive neuroscience research, followed by EEG and fMRI. Eye tracking data provided information about learner attributes and instructional aspects of a DLE. However, eye tracking cannot inform understanding of student engagement and emotion. Those were revealed through EEG. Yet, EEG data lack neural evidence of student engagement and cognitive processes. Educational studies involving fMRI can offer neural data that might be promising for student engagement and cognitive processes. Our discussion pointed out some of the key advances in our understanding of the brain cortical regions (e.g., inferior frontal gyrus) underlying learning and the potential to inform learning problems in DLEs. We conclude that studies in neuroscience using one tracking or imaging technique are inadequate to inform the underpinnings of learning and instruction in DLEs. We recommend that future studies consider their work in the light of data from multiple neuroscience techniques complementary to behavioural measures, to provide a more holistic understanding of learning and instruction in DLEs from a neuroscience perspective. Finally, we also suggest more collaboration among neurocognitive scientists, learning designers, educational 
researchers, and educators to enhance understanding in this area given that the findings of using neuroscience technique is still in infancy.

\section{References}

Anderson, O. R., Love, B. C., \& Tsai, M. J. (2014). Neuroscience perspectives for science and mathematics learning in technology-enhanced learning environments. International Journal of Science and Mathematics Education, 12(3), 467-474.

Ansari, D., \& Coch, D. (2006). Bridges over troubled waters: Education and cognitive neuroscience. Trends in Cognitive Sciences, 10(4), 146-151.

Ariasi, N., \& Mason, L. (2014). From covert processes to overt outcomes of refutation text reading: The interplay of science text structure and working memory capacity through eye fixations. International Journal of Science and Mathematics Education, 12(3), 493-523.

Bassett, D. S., \& Mattar, M. G. (2017). A network neuroscience of human learning: Potential to inform quantitative theories of brain and behavior. Trends in Cognitive Sciences, 21(4), 250-264.

Botvinick, M., \& Braver, T. (2015). Motivation and cognitive control: From behavior to neural mechanism. Psychology, 66(1), 83-113.

Boucheix, J. M., \& Lowe, R. K. (2010). An eye tracking comparison of external pointing cues and internal continuous cues in learning with complex animations. Learning and Instruction, 20(2), 123135.

Bryson, C., \& Hand, L. (2007). The role of engagement in inspiring teaching and learning. Innovations in Education \& Teaching International, 44(4), 349-362.

Canham, M., \& Hegarty, M. (2010). Effects of knowledge and display design on comprehension of complex graphics. Learning and Instruction, 20(2), 155-166.

Chang, H. Y., \& Linn, M. C. (2013). Scaffolding learning from molecular visualizations. Journal of Research in Science Teaching, 50(7), 858-886.

Chen, C. M., \& Wu, C. H. (2015). Effects of different video lecture types on sustained attention, emotion, cognitive load, and learning performance. Computers \& Education, 80, 108-121.

Chen, Y. C., \& Yang, F. Y. (2014). Probing the relationship between process of spatial problems solving and science learning: An eye tracking approach. International Journal of Science and Mathematics Education, 12(3), 579-603.

Dahlstrom, E., \& Bichsel, J. (2014). ECAR study of undergraduate students and information technology, 2014. Research report. Louisville, CO: ECAR, October 2014. Available from http://www.educause.edu/ecar.

Dahlstrom, E., Brooks, D. C., \& Bichsel, J. (2014). The current ecosystem of learning management 
systems in higher education: Student, faculty, and IT perspectives (p. 3). Research report. Louisville, CO: ECAR, September 2014. Available from http://www. educause.edu/ecar.

Diamond, A., \& Whitington, V. (2015). Studying early brain development: Educators' reports about their learning and its applications to early childhood policies and practices. Australasian Journal of Early Childhood, 40(3), 11-19.

Freitas, S. I., Morgan, J., \& Gibson, D. (2015). Will MOOCs transform learning and teaching in higher education? Engagement and course retention in online learning provision. British Journal of Educational Technology, 46(3), 455-471.

Gil, R., Virgili-Gomá, J., García, R., \& Mason, C. (2015). Emotions ontology for collaborative modelling and learning of emotional responses. Computers in Human Behavior, 51, 610-617.

Grabner, R. H., Ansari, D., Koschutnig, K., Reishofer, G., Ebner, F., \& Neuper, C. (2009). To retrieve or to calculate? Left angular gyrus mediates the retrieval of arithmetic facts during problem solving. Neuropsychologia, 47(2), 604-608.

Hames, E., \& Baker, M. (2013, October). EEG-based comparisons of performance on a mental rotation task between learning styles and gender. In Frontiers in Education Conference, 2013 IEEE (pp. 11761182). IEEE.

Henderson, J. M., Choi, W., Lowder, M. W., \& Ferreira, F. (2016). Language structure in the brain: A fixation-related fMRI study of syntactic surprisal in reading. NeuroImage, 132, 293-300.

Henrie, C. R., Halverson, L. R., \& Graham, C. R. (2015). Measuring student engagement in technology-mediated learning: A review. Computers \& Education, 90(1), 36-53.

Hockings, C., Cooke, S., Yamashita, H., McGinty, S., \& Bowl, M. (2008). Switched off? A study of disengagement among computing students at two universities. Research Papers in Education, 23(2), 191-201.

Jamet, E. (2014). An eye-tracking study of cueing effects in multimedia learning. Computers in Human Behavior, 32, 47-53.

Kanwal, J. S., Jung, Y. J., \& Zhang, M. (2015). Brain plasticity during adolescence: Effects of stress, sleep, sex and sounds on decision making. Anatomy \& Physiology, 6, e135.

Knowles, M. S. (1970). The modern practice of adult education: Andragogy versus earning and the learning organization: Examining the connection between the individual and the learning environment. Human Resource Development Quarterly, 9(4), 365-375.

König, W. A., Rädle, R., \& Reiterer, H. (2010). Interactive design of multimodal user interfaces. Journal on Multimodal User Interfaces, 3(3), 197-213.

Kozan, K., \& Richardson, J. C. (2014). Interrelationships between and among social, teaching, and cognitive presence. The Internet \& Higher Education, 21, 68-73. 
Lai, M. L., Tsai, M. J., Yang, F. Y., Hsu, C. Y., Liu, T. C., Lee, S. W. Y., ... \& Tsai, C. C. (2013). A review of using eye-tracking technology in exploring learning from 2000 to 2012. Educational Research Review, 10, 90-115.

Lee, W., \& Reeve, J. (2012). Self-determined, but not non-self-determined, motivation predicts activations in the anterior insular cortex: An fMRI study of personal agency. Social Cognitive and Affective Neuroscience, nss029.

Liu, N.-H., Chiang, C.-Y., \& Chu, H.-C. (2013). Recognizing the degree of human attention using EEG signals from mobile sensors. Sensors (Basel, Switzerland), 13(8), 10273-10286.

Liu, Y., Sourina, O., \& Nguyen, M. K. (2011). Real-time EEG-based emotion recognition and its applications. In Transactions on computational science XII (pp. 256-277). Springer Berlin Heidelberg.

Masson, S., Potvin, P., Riopel, M., Foisy, L. M. B., \& Lafortune, S. (2012). Using fMRI to study conceptual change: Why and how?. International Journal of Environmental \& Science Education, 7(1), 19-35.

Meyer, K., Rasch, T., \& Schnotz, W. (2010). Effects of animation's speed of presentation on perceptual processing and learning. Learning and Instruction, 20(2), 136-145.

Molina, A. I., Redondo, M. A., Lacave, C., \& Ortega, M. (2014). Assessing the effectiveness of new devices for accessing learning materials: An empirical analysis based on eye tracking and learner subjective perception. Computers in Human Behavior, 31, 475-490.

Montague, P. R., King-Casas, B., \& Cohen, J. D. (2006). Imaging valuation models in human choice. Annual Review of Neuroscience, 29, 417-448.

O'Flaherty, J., \& Phillips, C. (2015). The use of flipped classrooms in higher education: A scoping review. The Internet \& Higher Education, 25, 85-95.

Popa, L., Selejan, O., Scott, A., Mureşanu, D. F., Balea, M., \& Rafila, A. (2015). Reading beyond the glance: Eye tracking in neurosciences. Neurological Sciences, 36(5), 683-688.

Prat, C. S., \& Just, M. A. (2011). Exploring the neural dynamics underpinning individual differences in sentence comprehension. Cerebral Cortex, 21(8), 1747-1760.

Rayner, K. (2009). Eye movements and attention in reading, scene perception, and visual search. The Quarterly Journal of Experimental Psychology, 62(8), 1457-1506.

Richardson, D. C., \& Spivey, M. J. (2004). Eye tracking: Characteristics and methods. Encyclopedia of Biomaterials and Biomedical Engineering, 568-572.

Russell, J. A. (1983). Pancultural aspects of the human conceptual organization of emotions. Journal of Personality and Social Psychology, 45(6), 1281-1288. 
Säljö, R. (2010). Digital tools and challenges to institutional traditions of learning: technologies, social memory and the performative nature of learning. Journal of Computer Assisted Learning, 26(1), 5364.

Schmidt-Weigand, F., Kohnert, A., \& Glowalla, U. (2010). Explaining the modality and contiguity effects: New insights from investigating students' viewing behaviour. Applied Cognitive Psychology, 24(2), 226-237.

Shen, L., Wang, M., \& Shen, R. (2009). Affective e-learning: Using “emotional” data to improve learning in pervasive learning environment. Journal of Educational Technology \& Society, 12(2), 176189.

Siok, W. T., Perfetti, C. A., Jin, Z., \& Tan, L. H. (2004). Biological abnormality of impaired reading is constrained by culture. Nature, 431, 71-76.

Steinmetz, K. R. M., \& Atapattu, R. K. (2010). Meeting the challenge of preparing undergraduates for careers in cognitive neuroscience. Journal of Undergraduate Neuroscience Education, 9(1), A36.

Stewart, P. C. (2015). This is your brain on psychology wireless electroencephalography technology in a university classroom. Teaching of Psychology, 42(3), 234-241.

Stokes, S. P. (2001). Satisfaction of college students with the digital learning environment: Do learners' temperaments make a difference?. The Internet \& Higher Education, 4(1), 31-44.

Susac, A. N. A., Bubic, A., Kaponja, J., Planinic, M., \& Palmovic, M. (2014). Eye movements reveal students' strategies in simple equation solving. International Journal of Science and Mathematics Education, 12(3), 555-577.

Tang, Y., Zhang, W., Chen, K., Feng, S., Ji, Y., Shen, J., Reiman, E. M., \& Liu, Y. (2006). Arithmetic processing in the brain shaped by cultures. Proceedings of the National Academy of Science. USA, 103, 10775-10780.

Tsai, M. J., Hou, H. T., Lai, M. L., Liu, W. Y., \& Yang, F. Y. (2012). Visual attention for solving multiple-choice science problem: An eye-tracking analysis. Computers \& Education, 58(1), 375-385.

Varma, S., McCandliss, B. D., \& Schwartz, D. L. (2008). Scientific and pragmatic challenges for bridging education and neuroscience. Educational Researcher, 37(3), 140-152.

Wang, C. C., \& Hsu, M. C. (2014). An exploratory study using inexpensive electroencephalography (EEG) to understand flow experience in computer-based instruction. Information \& Management, 51(7), 912-923.

Weber, K., Christiansen, M., Petersson, K. M., Indefrey, P., \& Hagoort, P. (2016). fMRI syntactic and lexical repetition effects reveal the initial stages of learning a new language. The Journal of Neuroscience, 36(26), 6872-6880.

Weissberger, G. H., Gollan, T. H., Bondi, M. W., Clark, L. R., \& Wierenga, C. E. (2015). Language Journal of the Scholarship of Teaching and Learning, Vol. 18, No. 3, September 2018. josotl.indiana.edu 
and task switching in the bilingual brain: Bilinguals are staying, not switching, experts. Neuropsychologia, 66, 193-203.

Wu, J. Y. (2015). University students' motivated attention and use of regulation strategies on social media. Computers \& Education, 89, 75-90.

Yang, F. Y., Chang, C. Y., Chien, W. R., Chien, Y. T., \& Tseng, Y. H. (2013). Tracking learners' visual attention during a multimedia presentation in a real classroom. Computers \& Education, 62, 208-220.

Yoncheva, Y. N., Blau, V. C., Maurer, U., \& McCandliss, B. D. (2010). Attentional focus during learning impacts N170 ERP responses to an artificial script. Developmental Neuropsychology, 35(4), 423-445.

Zhao, J., Li, Q. L., Wang, J. J., Yang, Y., Deng, Y., \& Bi, H. Y. (2012). Neural basis of phonological processing in second language reading: An fMRI study of Chinese regularity effect. NeuroImage, 60(1), 419-425. 